\title{
Face Recognition: Some Challenges in Forensics
}

\author{
Anil K. Jain, Brendan Klare and Unsang Park \\ Michigan State University \\ East Lansing, MI, U.S.A \\ \{jain, klarebre, parkunsa\}@cse.msu.edu
}

\begin{abstract}
Face recognition has become a valuable and routine forensic tool used by criminal investigators. Compared to automated face recognition, forensic face recognition is more demanding because it must be able to handle facial images captured under non-ideal conditions and it has high liability for following legal procedures. This paper discusses recent developments in automated face recognition that impact the forensic face recognition community. Improvements in forensic face recognition through research in facial aging, facial marks, forensic sketch recognition, face recognition in video, nearinfrared face recognition, and use of soft biometrics will be discussed. Finally, current limitations and future research directions for face recognition in forensics are suggested.
\end{abstract}

Keywords- face recognition; forensics; facial marks; aging; forensic sketch; face in video; unconstrained face recognition

\section{INTRODUCTION}

Face recognition is the ability to establish a subject's identity based on his facial characteristics. Automatic face recognition has been extensively studied over the past two decades due to its important role in a number of application domains, including access control, visual surveillance, and deduplication of government issued identity documents (e.g., passport and driver license), to name a few. Face recognition systems generally operate under one of two scenarios: verification or identification [1]. In a verification scenario, the similarity between two face images is measured and a determination of either match or non-match is made. In an identification scenario, the similarity between a given face image (probe) and all the face images in a large database (gallery) is computed; the top (rank-1) match is returned as the hypothesized identity of the subject. Ideally, both of these scenarios are expected to operate in a "lights out" mode, i.e., the system makes an identity decision without requiring any human interaction.

The performance of automatic face recognition techniques has been evaluated in a series of tests conducted by the National Institute of Standards and Technology (NIST) using the FERET evaluation methodology [17]. The Face Recognition Vendor Test (FRVT) and Face Recognition Grand Challenge (FRGC) have continued these benchmarks with participants from both industry and academia. In the FRVT 2002 [13], an identification accuracy of $~ 70 \%$ was achieved for facial images with near frontal pose and normal lighting conditions on a large gallery (121,589 face images of 37,437 subjects). The most recent test, FRVT 2006 [14], involved a verification scenario; the best performing system showed a False Reject Rate (FRR) of 0.01 at a False Accept Rate (FAR) of 0.001 for high resolution (400 pixels between eyes) or 3D images. See Fig. 1 for examples of test images used in FRVT 2006.

Despite the impressive performance of automatic face recognition systems in a controlled setting, the benchmarked error rates in FRVT do not reflect the accuracy of face recognition systems when used in certain operational and forensic scenarios where it is not possible to make restrictive assumptions about ambient illumination, subject pose, sensor resolution, and compression (see Fig. 2). Contrary to the CSIeffect [27], which gives the illusory impression to citizens about the capabilities of state of the art face recognition technology, a number of prototype deployments (e.g., the Super Bowl game in Tampa in 2001 [33] and the Meinz railway station test in Germany in 2006 [36]) did not meet the required levels of matching accuracy. On the other hand, there are a few face biometric applications successfully deployed such as Smartgate in Australia [34] and the border control system between Hong Kong and China [35]), where user's cooperation is expected under a constrained environment. In addition to the effects of these extrinsic variables on face recognition accuracy, real-world forensic scenarios exhibit large intrinsic variations (e.g., due to facial aging, expression and cosmetic makeup) which further degrade the recognition performance and are generally not replicated in controlled studies.

Forensic science, or simply forensics, deals with the application of scientific principles to analyze data collected by law enforcement agencies. There is an increased emphasis on this field in order to prove or disprove the guilt of a suspect with high confidence under the legal system. Some examples of forensic science applications include blood spatter analysis, soil analysis, pathology, DNA identification, shoe print matching, latent fingerprint examination, and surveillance video analysis. These examples illustrate the range of data used in forensics, where one of the major goals is to establish the identity of the suspect. While fingerprint and DNA forensic identification are two of the most reliable and available identification methods in forensic science, continued progress in automated face recognition technology is necessary to improve the set of tools available to determine a person's identity, particularly from surveillance imagery. Such progress forensic face recognition is one of the goals of the FBI's Next Generation Identification program [52]. 


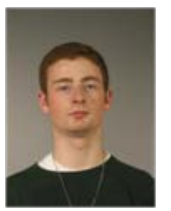

(a)

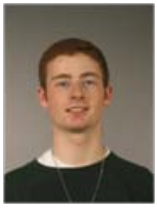

(b) (c)

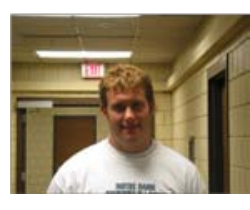

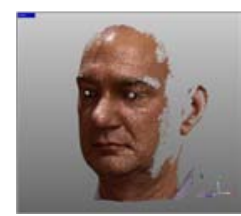

(d)
Figure 1. Example face images used in FRVT 2006 [14]. (a) Controlled lighting, neutral expression (IPD $=400$ pixels), (b) controlled lighting, smiling, (c) uncontrolled lighting, smiling (IPD = 190 pixels), and (d) 3D shape and texture. IPD stands for inter-pupillary distance.

Face recognition by humans has a long history in forensics. The first attempt to identify a subject by comparing a pair of facial photographs was reported in a British court in 1871 [11], and the first known systematic method for face recognition was developed by the French criminologist Alphonse Bertillon in 1882 [28].

The first paper on automatic face recognition appeared in 1966 by Bledsoe et al. [29]. The project was called "manmachine" because a set of facial features were extracted from the photographs by a human. These features were then fed to a computer to conduct automated matching. From the set of feature points (such as the center of pupils, inside and outside corners of eyes, point of widows peak, etc) a list of 20 distances were computed and used to measure the similarity between face images. The man-machine system was able to consistently outperform humans based on a database of over 2,000 photographs. Goldstein and Harmon [37] also used 22 descriptive features (morphological descriptions of the face, hair, eyebrows, etc.) to identify people based on face images. These features were provided to a set of trained jurors as well as computers to conduct identification tasks. Goldstein and Harmon concluded that six different features are required to identify a person in a database of 255 subjects, and predicted that 14 features are required to identify a person in a gallery of $4 \times 10^{6}$ faces.

The first fully automatic face identification system was developed by Kanade [38] using a set of facial parameters based on local histograms of gray scale pixel values. It was not until much later that many other automated face recognition systems were introduced. The Principal Component Analysis (PCA) method was first applied on face images by Sirovich [40]

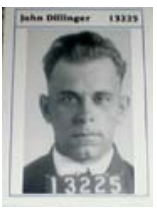

(a)

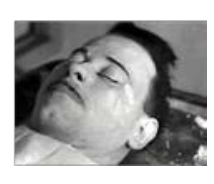

(b)

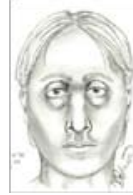

(c)

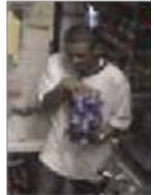

(d)

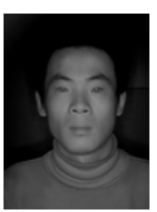

(e)
Figure 2. Examples of face images commonly encountered in forensic applications. (a) A mug shot, (b) an image of a deceased subject (John Dillinger), (c) a forensic sketch, (d) a CCTV frame, and (e) a near infrared (NIR) image
Table 1 . Summary of representative works in the history of automated face recognition research.

\begin{tabular}{|c|c|c|c|}
\hline & Approach & Database & $\begin{array}{c}\text { Identi- } \\
\text { fication } \\
\text { Accuracy }\end{array}$ \\
\hline $\begin{array}{c}\text { Bledsoe et al. } \\
\text { [29] }\end{array}$ & $\begin{array}{l}20 \text { features such as } \\
\text { width of mouth, width } \\
\text { of eyes, etc. (first semi- } \\
\text { automatic method.) }\end{array}$ & N/A* & N/A* \\
\hline $\begin{array}{c}\text { Goldstein and } \\
\text { Harmon [37] }\end{array}$ & $\begin{array}{l}22 \text { features including } \\
\text { simple morphological } \\
\text { description about face, } \\
\text { hair, eyebrows, etc. } \\
\text { (semi-automatic) }\end{array}$ & $\begin{array}{l}255 \text { images of } \\
7 \text { subjects }\end{array}$ & $53 \%$ \\
\hline Kanade [38] & $\begin{array}{l}\text { Local histogram (first } \\
\text { fully automatic } \\
\text { method) }\end{array}$ & $\begin{array}{l}20 \text { images of } \\
20 \text { subjects }\end{array}$ & $75 \%$ \\
\hline $\begin{array}{c}\text { Turk and } \\
\text { Pentland [41] }\end{array}$ & $\begin{array}{l}\text { Principal component } \\
\text { analysis (PCA or } \\
\text { Eigenface) }\end{array}$ & $\begin{array}{l}21,500 \text { images } \\
\text { of } 16 \text { subjects }\end{array}$ & $100 \%$ \\
\hline $\begin{array}{l}\text { Belhumeur et } \\
\text { al. [26] }\end{array}$ & $\begin{array}{l}\text { Linear Discriminant } \\
\text { Analysis (LDA) }\end{array}$ & $\begin{array}{l}160 \text { images of } \\
16 \text { subjects } \\
\text { (Yale DB) }\end{array}$ & $99.4 \%$ \\
\hline $\begin{array}{c}\text { Ahonen et al. } \\
\text { [43] }\end{array}$ & $\begin{array}{l}\text { Local Binary Patterns } \\
\text { (LBP) }\end{array}$ & $\begin{array}{l}\text { 1,196 Subjects } \\
\text { (FERET DB) }\end{array}$ & $97 \%$ \\
\hline
\end{tabular}

for image compression, then by Turk and Pentland [41] for identification. The ordered set of eigenvectors corresponds to a set of basis images that characterizes the variation between face images. PCA based approaches greatly reduced the computational burden and inspired more active research in face recognition. Another popular face recognition method is Linear Discriminant Analysis (LDA) [26], which is based on the Fisher's Liner Discriminant Analysis. The use of separate class labels for each subject in LDA provided better identification accuracy over PCA. Some other well known methods include Elastic Bunch Graph Matching (EBGM) [25] and Local Binary Pattern (LBP) [43] based feature representation. Table 1 shows a summary of representative works in face recognition.

While a majority of the face gallery images used in the forensics domain are mug shots (frontal pose and normal illumination with minimal expression), probe face images are often captured at different pose, illumination, resolution, and modality (e.g., infrared, video frames, etc.). For example, face images captured by surveillance cameras play a similar role as latent fingerprints, where the images present different degrees of difficulty in identification depending on motion blur, pose, and occlusion. With the rapid growth in the number of surveillance cameras worldwide (see Fig. 3), the progression of accurate and robust face identification techniques in videos is of utmost importance to law enforcement agencies. Face images appearing in faxed, printed, and scanned documents are also considered in the forensics domain [49]. 


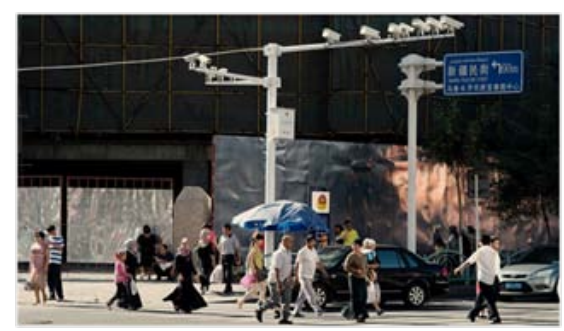

(a)

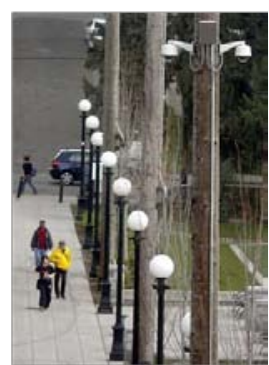

(b)
Figure 3. Surveillance cameras on the streets in China [50] [51]. There are about 400,000 surveillance cameras in Beijing alone that provide $100 \%$ coverage of public places (schools, hospitals, subways, etc.).

Forensic face recognition departs from automated face recognition in that it generally includes a human in the loop (Fig. 4). Many factors contribute to this requirement, such as low quality probe images, the use of metadata (demographics) to improve the chance of a successful match, and the need to present sound evidence in courts of law. The typical forensic face recognition scenario begins with a large gallery of face images, such as mug shot images and driver license photographs. For example, the face recognition system at the Pinellas County Sherriff's Office has a database of over 6 million face images [3], which is populated by both mug shot images (captured at the time of arrest) and Florida Department of Motor Vehicles (DMV) images [2]. An automatic face recognition system is needed to enable queries against such a large gallery database. Given that the queries in the forensic scenarios are often captured under non-ideal situations (e.g., off-pose and low resolution CCTV frames, images captured from an ATM, a forensic sketch, or an image from a social networking site), the most similar $N$ subjects (top $N$ ranks) retrieved from the automatic system are considered "soft" suspects, which are then manually examined by forensic experts to determine the correct match. The manual inspection procedure needs to be standardized to minimize the subjective decisions. The National Academies report on "Identifying the Needs of the Forensic Sciences Community" made thirteen specific recommendations [12], which also emphasize the need for standardization of the inspection and interpretation of forensic evidence and performance measurement.

Note that a high match score between two face images alone may not be sufficient for a conviction in criminal court. Instead, investigators use the face recognition to identify a set of candidates; additional cues or supporting evidence from other sources is used to find the most likely suspect to the crime.

In summary, the following characteristics distinguish forensic face recognition from automatic face recognition:

1. Probe image quality is non-ideal (e.g. partial face, offpose, high compression, and low resolution)

2. Top $N$ matches are examined, as opposed to a rank-1 match

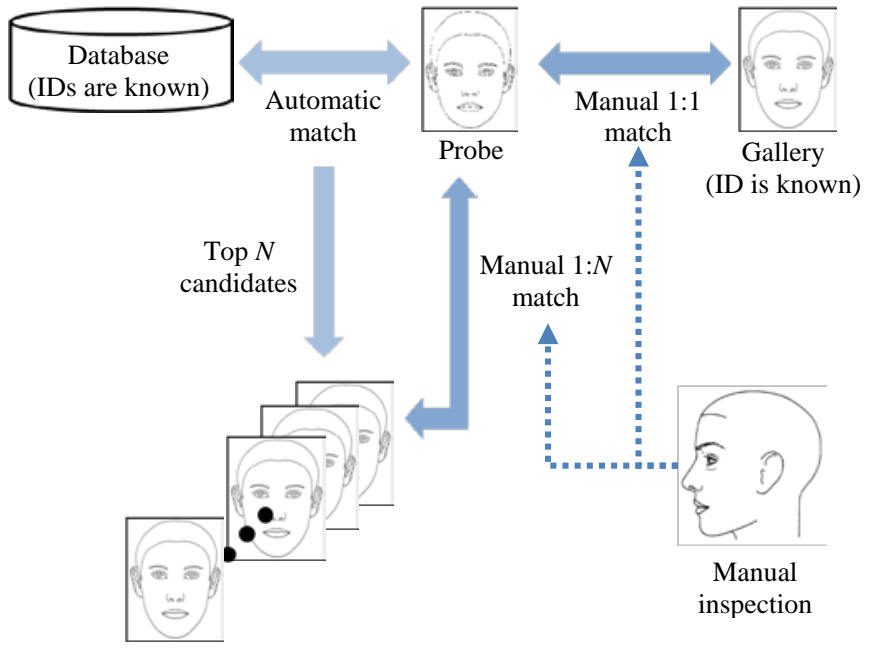

Figure 4. Schematic of forensic face recognition process. While the inspection procedure is fully manual when comparing two images, it is semiautomatic in searching a large database. In many scenarios, forensic face recognition is not yet fully automatic.

This paper is meant to increase the awareness and understanding of important challenges in forensic face recognition. We provide some of the major research topics in forensic face recognition, including age invariant face recognition, facial mark based matching and retrieval, matching forensic sketches to mug shots, face recognition in surveillance videos (CCTV, ATM feed, etc.), and matching near infrared images to photographs.

\section{Forensic FACE RECOGNITION CHALLENGES}

This section will discuss advances in several face recognition research areas of importance to forensic face recognition. The choice of these specific problems is influenced by our own ongoing research.

\section{A. Facial Aging}

Many face recognition scenarios exhibit a significant age

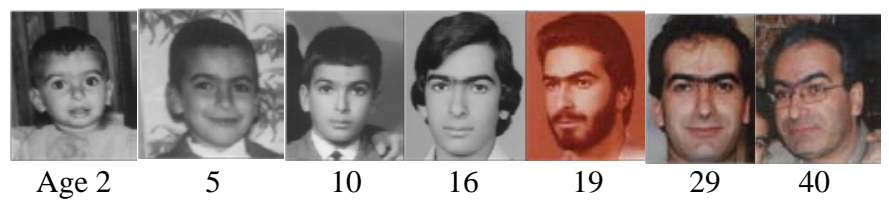

(a) Images of a subject from the FG-NET database [7]

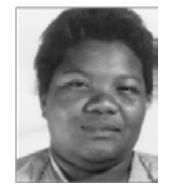

Age 25

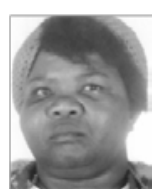

36

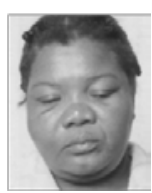

40

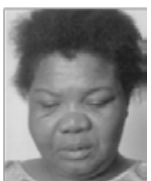

43

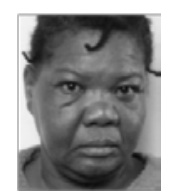

48 (b) Images of a subject from the MORPH database [35]

Figure 5. Change in facial appearance due to aging. 


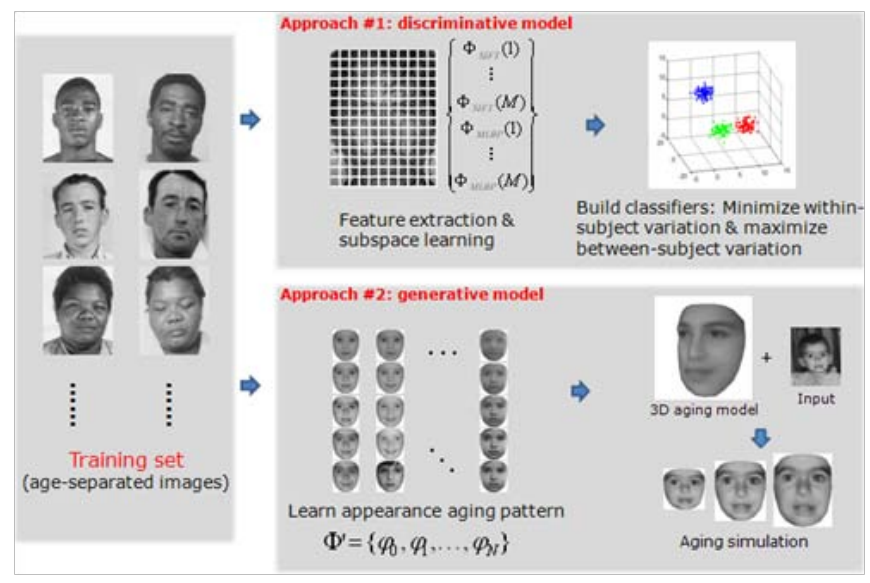

Figure 6. Schematic of discriminative and generative aging models.

difference between the probe and gallery images of a subject. As the age between a probe and a gallery image of the same subject increases, the accuracy of state of the art face recognition system generally decreases. Some face recognition applications where such age differences are encountered include 1) identifying missing children and 2) detecting if a user/suspect is present in a database (such as law enforcement or DMV databases).

Facial aging is a complex process that affects both the shape and texture (e.g., skin tone or wrinkles) of a face. This aging process also appears in different manifestations in different age groups. While facial aging is primarily represented by facial growth in younger age groups $(\leq 18$ years), it is mostly characterized by relatively large texture changes and minor shape changes (e.g., due to change in subject's weight or stiffness of skin) in older age groups ( $>18$ years). Fig. 5 shows aging variations of two subjects in the FG-NET [7] and MORPH [31] databases. As expected, the facial appearance changes more drastically at younger ages. In addition to facial aging, there are other factors that influence facial appearance as well (e.g. pose, lighting, expression, occlusion) which makes it difficult to study the aging pattern using these two public domain longitudinal face databases.

$\mathrm{Li}$ et al. [32] proposed both discriminative and generative aging models for age invariant face recognition. The generative model learns the parametric aging model in the 3D domain to generate synthetic images and reduce the age gap between probe and gallery. The generative 3D aging technique uses a pose correction method and an aging model in the 3D domain. 3D modeling is well suited to capture the aging patterns due to the 3D nature of aging. Because no 3D aging database is currently available, the proposed 3D aging model was built using a 2D face aging database. The discriminative model learns the salient features to better recognize the face images across age gaps. Fig. 6 shows the schematic of both the generative and discriminative aging modeling methods. Fig. 7 shows example matching results where aging modeling improved the matching accuracy of a leading face recognition

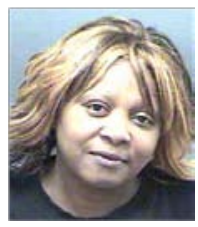

age 51

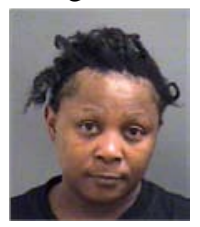

age 41

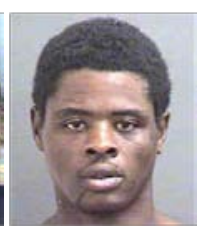

40

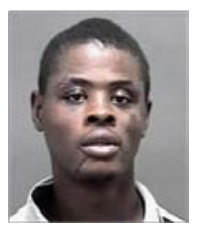

34

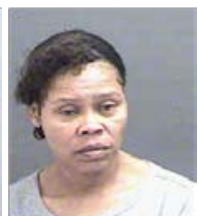

42

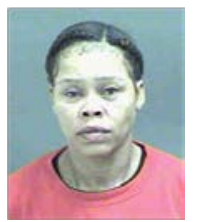

41

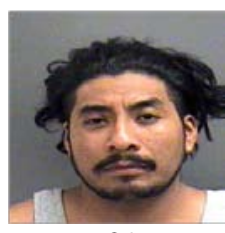

29

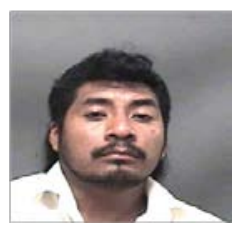

23
Figure 7. Example of face recognition in the presence of aging where a commercial matcher fails but the proposed aging model succeeds [36]. The top row shows the gallery images, and the bottom row shows the probe images of the same subjects.

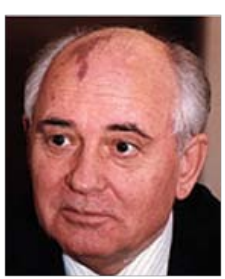

(a)

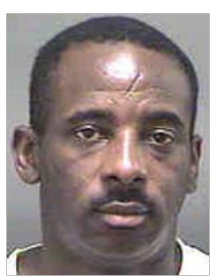

(b)

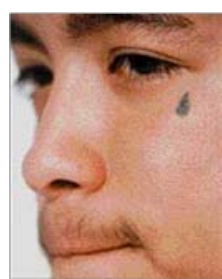

(c)
Figure 8. Example face images with distinctive facial marks. (a) Large birth mark, (b) scar, and (c) "tear drop” tattoo.

engine, FaceVACS [21]. A face recognition test with 10,000 images of 10,000 subjects from MORPH database in the probe and gallery showed approximately an $8 \%$ improvement in rank-1 identification accuracy by using the proposed aging models [32].

\section{B. Facial Marks}

Local facial mark features such scars, moles, and freckles play an important role for matching face images in forensic applications (see Fig. 8) [22]. The explicit use of face marks has become valuable due to the availability of higher resolution sensors, compatibility with manual identification, and the growing size of face image databases. Local facial mark features provide a unique capability to investigate, annotate, and exploit face images in forensic applications by improving both the accuracy and the matching speed of facerecognition systems. This information is also necessary for forensic experts to give testimony in courts of law where they are expected to conclusively identify suspects [23].

Most of the current photo-based identifications in law enforcement units and related security organizations involve a manual verification stage. The identification information is often provided by a victim or witness in terms of verbal descriptions and hand-drawn sketches [6]. Spaun [22] [23] describes the facial examination process carried out in the law 


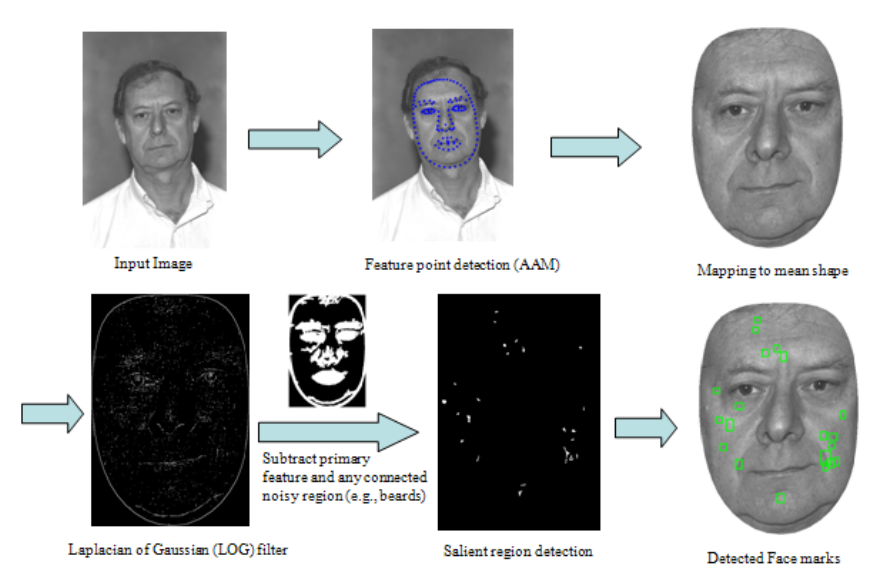

Figure 9. Schematic of automatic mark detection process [5].

enforcement agencies (the five-step manual examination procedure is referred to as ACE-V [24]), where one of the major steps is to identify the "class" and "individual" characteristics. The class information includes overall facial shape, hair color, presence of facial hair, shape of the nose, presence of freckles, etc. The individual characteristics include the number and locations of freckles, scars, tattoos, chipped teeth, lip creases, number and location of wrinkles, etc., in a face. An automatic procedure for this examination will not only reduce the time-consuming and subjective manual process, but is likely to be more consistent and accurate. Further, it is expected that the computer-aided automatic feature extraction and representation will help standardize the examination process and make the process more efficient.

Conventional face-recognition systems typically encode the face images by utilizing either local or global texture features. However, these techniques do not explicitly utilize local marks (e.g., scars and moles) and usually expect the input to be a full face image. To fill this void, Park and Jain proposed the automatic facial mark detection process shown in Fig. 9 [5].

Face mark patterns have been shown to be reliable when used as a soft biometric [5]. Park and Jain developed a framework for text query retrieval of subjects using face marks [5]. Textual queries allow investigators to retrieve a list of subjects who contain some combination of face marks, such as "Mole on Left Cheek" AND "Scar on Forehead".

A face mark retrieval system is not expected to identify a subject uniquely. Instead, it can be used to filter a candidate population. A witness can provide the information that a suspect has a mole on his left cheek and a scar on his forehead. Even in scenarios where no probe face image exists, this information can still be leveraged in an automated fashion to (for example) retrieve a list of felons who meet this criterion. Coupled with additional demographic information that may be available (gender, age, height, etc.), investigators may be left with only a few dozen individuals to investigate.

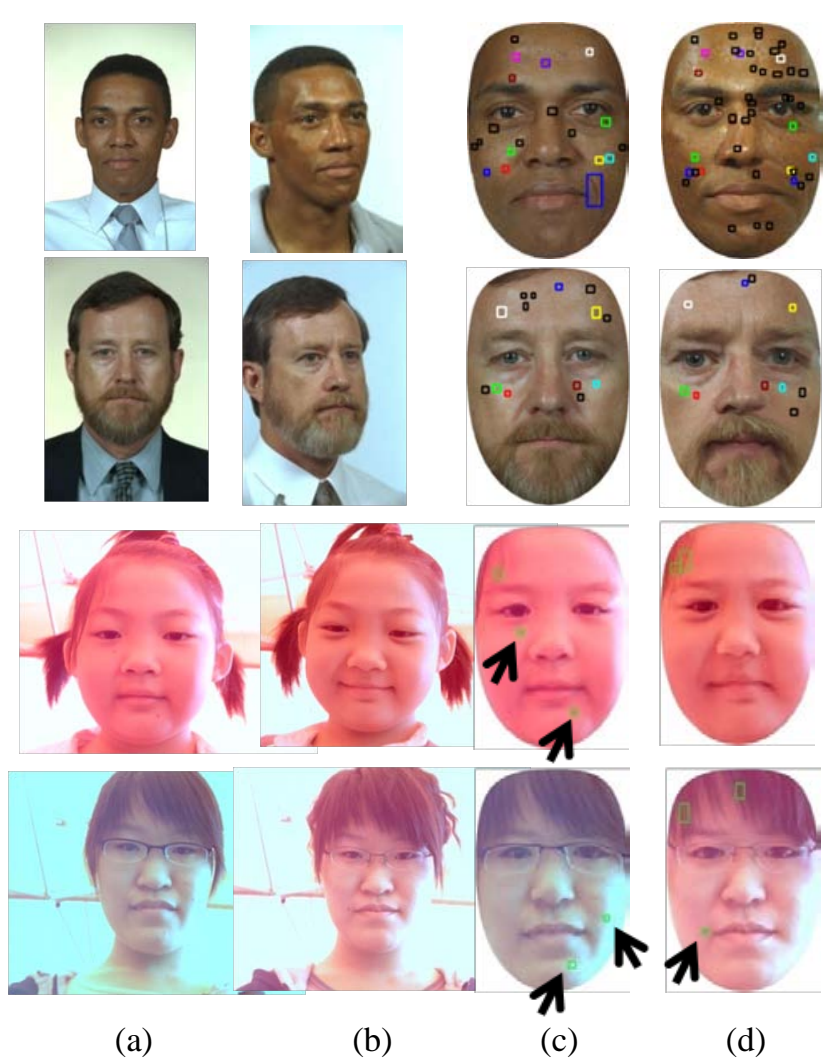

Figure 10. Example image pairs where facial marks helped to improve the matching performance. (a) (b) Probe and gallery images for the first two rows and two images of identical twins for the third and fourth rows. (c) (d) Facial mark detection results from (a) and (b).

In addition to permitting face retrieval without a face image, Fig. 10 shows a few example image pairs where the explicit use of facial marks improved the matching accuracy (when fused with a commercial matcher). The first and second rows in Fig. 10 show example images that did not match at rank-1, but when fused with face mark match scores they were correctly matched at rank-1. The third and fourth rows show facial images of identical twins that were incorrectly matched at rank-1 unless face marks were used. Face marks are critical in the identification of identical twins [9]. The use of facial mark improved the rank-1 identification accuracy of FaceVACS by about $0.5 \%$ based on 213 images of 213 subjects as probes and 10,213 images of 10,213 subjects in the gallery [5].

\section{Forensic Sketch Recognition}

When no photograph of a suspect is available, a forensic sketch is often generated. Forensic sketches are an artist rendition of a person's facial appearance that is derived from an eye witness description. Forensic sketches have a long history of use, where traditionally they have been disseminated to media outlets and law enforcement agencies in the hopes that someone will recognize the person in the sketch. Forensic sketches can be misleading due to errors in witness memory recall that cause inaccuracies in the sketch drawn by a forensic artist. Because a significant amount of time is needed to 

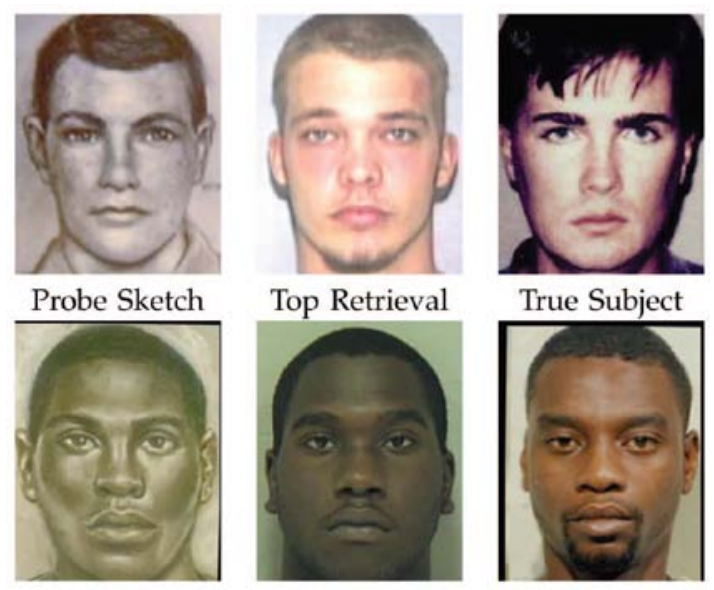

Figure 11. Examples of failed matches in forensic sketch recognition [6]. Images from [18].

generate a single forensic sketch, they generally represent culprits who committed the most heinous crimes (e.g. murder and sexual assault). Thus, the ability to match forensic sketches to mug shot databases is of great importance.

Commercial face recognition systems are not designed to match forensic sketches against face photographs. To fill this gap, Klare et al. [6] developed a framework for matching forensic sketches to photographs. Using a forensic sketch, the proposed system allows investigators to match the sketch against large face databases. The method from [6] encodes the structure of both sketches and photographs using local binary patterns [15] and SIFT feature descriptors [16]. Each of these two feature descriptors has the desirable property of having little variation between the sketch and photo modalities. With sketch and photos represented using feature descriptors, multiple linear discriminant subspaces are learned on vertical slices of face image patches in a framework called Local Feature-based Discriminant Analysis (LFDA). LFDA demonstrated substantial improvements over a commercial recognition system [6]. For example, when using 49 probe subjects and 10,159 gallery subjects, the rank-50 accuracy of LFDA with race and gender filtering was $44.9 \%$. This is compared to a rank-50 accuracy of $26.53 \%$ for FaceVACS with race and gender filtering. The use of specially designed sketch recognition systems such as LFDA is necessary to maximize the capabilities of forensic sketch recognition.

A major difficulty in forensic sketch recognition is a witness's inability to correctly remember the appearance of the subject. Fig. 11 shows two examples where the top retrieved photograph (second column) is incorrect, but more closely resembles the subject in the sketch (first column) than the true photo of the subject (third column). This problem also extends beyond automated sketch recognition (i.e., humans must also overcome the same noise), and it demonstrates the difficulty of forensic sketch recognition.

\section{Face Recognition in Video}

Face recognition in video has gained importance due to the widespread deployment of surveillance cameras. The ability to

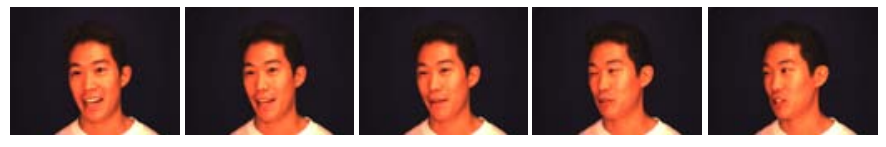

(a)
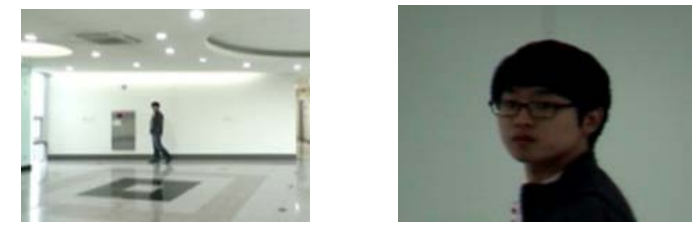

(b)

Figure 12. Example face images in video. (a) Five consecutive frames in a video. (b) Global and close-up views captured by a pair of static and PTZ cameras.

automatically recognize faces in video streams will facilitate a method of human identification using the existing networks of surveillance cameras. However, face images in video often contain non-frontal poses of the face and may undergo severe lighting changes. Fig. 12 (a) shows example frames captured in a video stream [53]. The multiple frames captured from the same subject in a video stream enable the system to selectively use face images with the best quality (e.g., frontal pose or neutral expression). Temporal information, such as the dynamic facial expression changes, can also be used in face recognition [48]. Since most of the face images captured from conventional surveillance cameras appear at low resolution, a pair of static and PTZ camera systems may be used in tandem. For example, Fig. 12 (b) shows both global and close-up view images captured by a pair of static and PTZ camera system developed for the task of face recognition at a distance [44]. The static camera alerts the PTZ camera of the presence of a subject, and the PTZ camera then acquires a higher quality face image of the subject.

Methods also exist to overcome many of the pose and lighting variations in video. For example, Volker and Blanz [30] proposed a view-synthesis method by using a 3D morphable model to synthesize a 3D face model from a 2D image. This method only requires a single image at an arbitrary pose, but the fitting process takes a few minutes and requires manual initial alignment. Park and Jain [4] used the structurefrom-motion method which utilizes facial landmarks obtained from video sequences to infer the 3-D face shape. The use of 3D modeling and a PTZ camera improved the rank-1 identification accuracy by about $40 \%$ and $98 \%$, respectively. The 3D modeling face recognition experiments used the CMU Face In Action (FIA) database [53] with 221 subjects, and the PTZ camera experiments used a combination of a private database and the MORPH database with 20 probe subjects and 10,020 gallery subjects.

\section{E. Near-Infrared Face Recognition}

The use of near-infrared (NIR) face images has been proposed as a method for overcoming the impact of varying illumination [45] [46]. Similar to sketch recognition, most applications of NIR face recognition are in a heterogeneous 


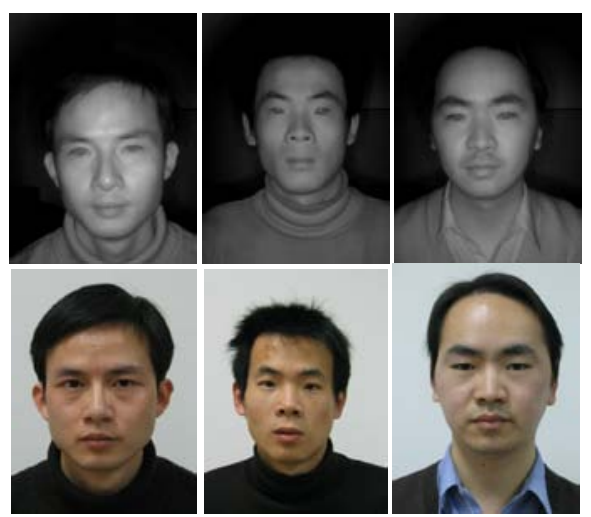

Figure 13. Near infrared face images (first row), and the corresponding photographs (second row). Near infrared face images have ideal properties for acquiring face images in surveillance scenarios. Images are from [47].

face recognition scenario, where the gallery images are standard photographs. An example of face images acquired in NIR and visible (VIS) spectrums can be found in Fig. 13.

Klare and Jain [45] proposed a feature-based framework for matching NIR face probe images to visible gallery images. Both NIR and VIS face images were represented using SIFT and LBP descriptors. A random subspace framework was used with a sparse representation matcher. Using the public HFB dataset [47], Klare and Jain's proposed method when combined with a commercial matcher, FaceVACS [21], achieved a true accept rates of $\sim 94 \%$ at a false accept rate of $1.0 \%$.

Given the high accuracy of matching NIR and VIS face images, surveillance systems should also consider the use of NIR cameras to compensate for illumination issues. Because the human eye is not sensitive to NIR illumination, NIR camera systems can covertly utilize directed NIR illumination without alerting subjects. This advantage allows the illumination to be tailored for improved face recognition in a particular environment.

\section{F. Soft Biometrics}

Systems designed to leverage soft biometrics that may be determined using face images are another useful tool in forensic face recognition. Soft biometric demographic information (such as race, gender, and general age) can usually be determined from low quality face images. The explicit use of this information in difficult recognition scenarios has been shown to improve face recognition accuracies [5] [6] [9]. Another example of soft biometrics in forensics is the use of tattoos for suspect and victim identification [55].

\section{SUMMARY AND FUTURE WORK}

Despite the host of tools highlighted in the previous section, many limitations still exist in forensic face recognition.

\section{A. Individuality Models}

Face recognition systems return a match score between two face images indicating their level of similarity. Often these scores are normalized to the range $[0,1]$, making them analogous to a probability of a true match. However, these match scores do not truly measure such a probability because no face individuality models have been developed.

Spaun [22] mentioned how the lack of face individuality models limits legal testimony to being opinion-based. A recent study on forensic facial comparison similarly mentioned this shortcoming [8]. Klare and Jain [9] proposed an organization of the salient information contained in facial photographs into three feature levels (analogous to the three levels in fingerprint features). By organizing the information contained in facial images, the taxonomy of facial features can facilitate studies on face individuality.

Despite initial efforts in [5] [8] [9] [55], we are still far removed from having a valid individuality model. Until such an individuality model is developed and accepted in a peer reviewed setting, the automated face recognition results have limited use as evidence in court. Thus, the substantial activity in face recognition research is greatly limited by a relatively non-existent amount of research in facial individuality studies. It is critical for this gap to be closed.

\section{B. Component-based Recognition}

Face recognition systems are generally designed to match images of full faces. Forensic scenarios exist in which a component-based recognition system would be useful. Such a system would receive as input a specific sub-region of the face containing components such as a nose, mouth, or eyebrows. However, with the exception of perioccular recognition systems [10], few advances have been made in component based recognition.

A component-based COTS FR system would ideally have the following functionality to augment forensic capabilities. Given a probe face (partial or complete), matching and retrieval would be performed for each facial component (eyes, nose, mouth, chin, and eyebrows). While such a system would have limited benefit in standard face recognition scenarios, it would be of great value to forensic investigators. Allowing investigators to specify a particular region of the face prevents incomplete, noisy, and missing regions from degrading the recognition accuracy. Further, a better understanding of component-based face recognition should facilitate the study of individuality models.

\section{CONCLUSIONS}

This paper highlights some of the problems and challenges in the field of forensic face recognition. Contrary to standard automated face recognition, forensic face recognition offers a set of tools that can help investigators narrow the identity of a subject, but not fully perform the identification. There has been a substantial improvement in the capabilities of forensic face recognition as a result of ongoing studies on facial aging, facial marks, sketch to photo matching, video based face recognition, and NIR image to photo match. However, many challenging problems related to forensic face recognition still exist, which offer excellent opportunities to face recognition researchers.

\section{ACKNOWLEDGMENT}

Part of Anil Jain's research was supported by WCU (World Class University) program through the National Research 
Foundation of Korea funded by the Ministry of Education, Science and Technology (R31-10008).

\section{REFERENCES}

[1] A. K. Jain, P. Flynn, A. Ross (Eds), Handbook of Biometrics, Springer, 2007

[2] S.Z. Li and A.K. Jain (eds.), Handbook of Face Recognition, Springer, 2005

[3] PCSO “Agency's Use Of Driver Licenses In Facial Recognition Is First In The Nation," The Star, Vol. 21, No. 3. 2009.

[4] U. Park and A.K.Jain, "3D Model-Based Face Recognition in Video," Proc. ICB, 2007.

[5] U. Park and A. K. Jain, "Face Matching and Retrieval Using Soft Biometrics,” IEEE Trans. on IFS, Vol. 5, No. 3, pp. 406-415, 2010

[6] B. Klare, Z. Li, and A. K. Jain, "Matching Forensic Sketches to Mugshot Photos," IEEE Trans. on PAMI, 2011. To appear.

[7] FG-NET Aging Database, http://www.fgnet.rsunit.com, 2010.

[8] M. P. Evison and R.W. Vorder Bruegge (Eds.), Computer-aided Forensic Facial Comparison. CRC Press, 2010, ch. 1,10.

[9] B. Klare and A. K. Jain, "On a Taxonomy of Facial Features," Proc. Biometrics: Theory, Applications and Systems (BTAS), 2010.

[10] U. Park, R. Jillela, A. Ross, and A. K. Jain, "Periocular Biometrics in the Visible Spectrum,” IEEE Trans. on IFS, 2011. To appear.

[11] G. Porter and G. Doran, “An Anatomical and Photographic Technique for Forensic Facial Identification,” Forensic Science International, Vol. 114, pp. 97-105, 2000.

[12] National Research Council, "Committee on Identifying the Needs of the Forensic Sciences Community,” 2009.

[13] P. J. Phillips et al., "Face Recognition Vendor Test 2002: Evaluation Report,” Tech. Report NISTIR 6965, NIST, 2003

[14] P. J. Phillips et al., "Face Recognition Vendor Test 2006: FRVT 2006 and ICE 2006 Large-Scale Results,” Tech. Report NISTIR 7408, 2007

[15] T. Ojala, M. Pietikainen, and T. Maenpaa, "Multiresolution Gray-Scale and Rotation Invariant Texture Classification with Local Binary Patterns,” IEEE Trans. on PAMI, vol. 24, no. 7, pp. 971-987, July 2002

[16] D. Lowe, "Distinctive Image Features from Scale-Invariant Keypoints," Int'l J. Computer Vision, vol. 60, no. 2, pp. 91-110, 2004.

[17] P.J. Phillips, H. Moon, S. Rizvi, and P. Rauss, "The FERET Evaluation Methodology for Face-Recognition Algorithms," IEEE Trans. on PAMI, vol. 22, no. 10, pp. 1090-1104, Oct. 2000.

[18] L. Gibson, Forensic Art Essentials. Elsevier, 2008.

[19] H. Ling, S. Soatto, N. Ramanathan, and D. Jacobs, "A Study of Face Recognition as People Age,” Proc. IEEE Int’l Conf. Computer Vision, pp. 1-8, 2007.

[20] N. Ramanathan and R. Chellappa, "Face Verification Across Age Progression,” Proc. IEEE CS Conf. Computer Vision and Pattern Recognition, vol. 2, pp. 462-469, 2005.

[21] FaceVACS Software Developer Kit, Cognitec Systems GmbH, http://www.cognitec-systems.de, 2010.

[22] N. A. Spaun, "Forensic Biometrics from Images and Video at the Federal Bureau of Investigation,” in Proc. BTAS, 2007, pp. 1-3.

[23] N. A. Spaun, "Facial Comparisons by Subject Matter Experts: Their Role in Biometrics and Their Training," in Proc. ICB, 2009, pp. 161168.

[24] H. Tuthill and G. George, Individualization: Principles and Procedures in Criminalistics. Lightning Powder Company, Inc., 2002.

[25] L. Wiskott, J.-M. Fellous, N. Kruger, and C. von der Malsburg, "Face Recognition by Elastic Bunch Graph Matching,” IEEE Trans. on PAMI, vol. 19, no. 7, pp. 775-779, Jul. 1997.

[26] P. N. Belhumeur, J. P. Hespanha, and D. J. Kriegman, "Eigenfaces vs. Fisherfaces: Recognition Using Class Specific Linear Projection,” IEEE Trans. on PAMI, vol. 19, no. 7, pp. 711-720, Jul. 1997.

[27] M.Houck, “CSI: The Reality,” Scientific American, pp 85-89, June 2006.
[28] A. Bertillon, Signaletic Instructions including the theory and practice of Anthropometrical Identification, R.W. McClaughry Translation. The Werner Company, 1896.

[29] W. W. Bledsoe, “Man-machine Facial Recognition,” Tech. Report. PRI: 22, Panoramic Res. Inc., Palo Alto, CA, 1966.

[30] V. Blanz and T. Vetter, "Face Recognition Based on Fitting a 3D Morphable Model,” IEEE Trans. on PAMI, pp. 1063-1074, 2003

[31] K. Ricanek Jr. and T. Tesafaye, "Morph: A Longitudinal Image Database of Normal Adult Age-Progression,” Proc. Int'l Conf. Automatic Face and Gesture Recognition, pp. 341-345, 2006.

[32] Z. Li, U. Park, and A. K. Jain, "A Discriminative Model for Age Invariant Face Recognition,” IEEE Trans. on IFS, Under review.

[33] J. Bonner, "Looking for Faces in the Super Bowl Crowd," Access Control \& Security Systems Magazine, 2001.

[34] Smartgate, http://www.customs.gov.au/site/page5552.asp

[35] Chinese/Hong Kong border automated with biometrics, Biometric Technology Today, vol. 15, no. 5, pp. 3, 2007.

[36] J. S. Dempsey and L. S. Forst, An Introduction to Policing (5th ed.), Delmar-Cengage Learning Publishers, NY, 2010.

[37] A. J. Goldstein and L. D. Harmon, "Identification of Human Faces," Proceedings of IEEE, vol. 59, no. 5, pp. 748-760, 1971.

[38] T. Kanade, "Picture Processing System by Computer Complex and Recognition of Human faces," Ph.D. dissertation, Kyoto University, 1973.

[39] R. Brunelli and T. Poggio, "Face Recognition: Features versus Templates,” IEEE Trans. on PAMI, vol. 15, no. 10, pp. 1042-1052, 1993.

[40] L. Sirovich and M. Kirby, "Low-dimensional Procedure for the Characterization of Human Faces," Journal of Optical Society of America, A, vol. 4, no. 3, pp. 519-524, 1987.

[41] M. Turk and A. Pentland, "Eigenfaces for Recognition," Cognitive Neuroscience, vol. 3, pp. 72-86, 1991.

[42] P. N. Belhumeur, J. P. Hespanha, and D. J. Kriegman, "Eigenfaces vs. Fsherfaces: Recognition Using Class Specific Linear Projection,” IEEE Trans. on PAMI, vol. 19, no. 7, pp. 711-720, 1997.

[43] T. Ahonen, A. Hadid, and M. Pietikainen, "Face Description with Local Binary Patterns: Application to Face Recognition,” IEEE Trans. on PAMI, vol.28, no.12, pp.2037-2041, 2006.

[44] H.-C. Choi, U. Park, and A. K. Jain, "PTZ Camera Assisted Face Acquisition, Tracking \& Recognition,” Proc. BTAS, 2010

[45] B. Klare and A. K. Jain, "Heterogeneous Face Recognition: Matching NIR to Visible Light Images,” Proc. ICPR, 2010.

[46] S. Liao et al., "Heterogeneous face recognition from local structures of normalized appearance," Proc. ICB, 2009.

[47] S. Li, Z. Lei, and M. Ao. "The HFB face database for heterogeneous face biometrics research,” Proc. CVPRW, 2009.

[48] N. Ye and T. Sim, "Towards general motion-based face recognition," Proc. CVPR, pp. 2598 - 2605, 2010.

[49] Thirimachos Bourlai, Arun Ross, and Anil K. Jain, "Restoring Degraded Face Images: A Case Study in Matching Faxed, Printed and Scanned Photos," IEEE Trans. on IFS, 2011. To appear.

[50] http://www.sott.net/image/image/s1/36913/full/002564baf2f90d3f2b193 e.jpg

[51] http://seattletimes.newsource.com/ABPub/2008/04/21/2004364541.jpg

[52] “Next Generation Identification,” http://www.fbi.gov/about-us/cjis/fin gerprints_biometrics/ngi/ngi2

[53] R. Goh, L. Liu, X. Liu, and T. Chen., "The CMU Face In Action (FIA) Database, ” Proc. Analysis and Modeling of Faces and Gestures, 2005.

[54] A. K. Jain, J.-E. Lee, R. Jin, N. Gregg, “Content Based Image Retrieval: An Application to Tattoo Images,” Proc. ICIP, 2009

[55] M. P. Evison and R.W. Vorder Bruegge, Eds., "Computer-aided forensic facial comparison.," CRC Press, 2010. 